Journal of

Accident and

Emergency

Medicine 1994

11, 79-84
Correspondence:

K. Barrett,

Department of

Psychiatry, School of

Postgraduate

Medicine,

Thornburrow Drive, Hartshill, Stoke-on-

Trent, ST4 7QB

\title{
Sequelae of minor head injury: the natural history of post-concussive symptoms and their relationship to loss of consciousness and follow-up
}

\author{
K. BARRETT, ${ }^{1}$ A.B.WARD, ${ }^{2}$ A. BOUGHEY, ${ }^{1}$ M. JONES $^{3}$ \& W. MYCHALKIW ${ }^{4}$ \\ ${ }^{1}$ Department of Psychiatry, School of Postgraduate Medicine, Hartshill, Stoke-on-Trent, \\ ${ }^{2}$ Department of Rehabilitation Medicine, Haywood Hospital, Burslem, Stoke-on-Trent, \\ ${ }^{3}$ Department of Mathematics, Keele University, Staffordshire and \\ ${ }^{4}$ Department of Clinical Psychology, North Staffordshire Hospital Centre, Hartshill, Stoke-on-Trent
}

\section{SUMMARY}

The character and natural history of postconcussive symptoms were studied in two subject groups: patients admitted to hospital for observation following brief loss of consciousness (LOC) as a result of head injury and patients who attended the accident unit after head injury but not were not admitted. Follow-up data were obtained from the hospitalized group at standardized out-patient interview and from the non-hospitalized group by postal questionnaire. Two- and 12-week data are presented on 24 hospitalized and 58 non-hospitalized patients. The type and frequency of symptoms was similar in the two groups and rank ordering was significantly correlated. However, the reduction in symptom scores (number of symptoms) 12 weeks post-injury was significantly greater in the hospitalized than the non-hospitalized group. The possible significance of this is discussed.

Key words: follow-up, head injury, loss of consciousness, post-concussive symptoms

\section{INTRODUCTION}

This paper examines the natural history of postconcussive symptoms over a period of 3 months following minor head injury. Up to $1 \%$ of the population of the United Kingdom attend accident and emergency (A\&E) departments each year as a result of head injury. At least $75 \%$ of these injuries are minor and only a small proportion of patients who are considered at risk of intracranial haemorrhage are admitted for overnight observation. Patients not admitted are given written instructions listing the symptoms which should, if they develop, lead the patient to return to hospital. Neither group receive out-patient follow-up routinely as post-concussive symptoms are generally regarded as universal, mild and short-lived. There have been a number of followup studies which contradict the view that such symptoms are invariably benign. ${ }^{1-4}$ These have not, to date, altered clinical practice. This may result, in part, from the long held, if poorly substantiated view that patients with persisting and disabling symptoms after minor head injury are at best neurotic and at worst compensation-seeking malingerers.

The changes that occurred in post-concussive symptoms in the 3 months following minor head injury were examined, contrasting patients admitted to hospital overnight with patients not admitted. The study took place in the context of an experimental service development in North Staffordshire. The health authority serves a population of 470000 with a $70 \%$ urban and a $30 \%$ rural population. The A\&E Department is within a 1000 -bed hospital centre in the city of Stoke-on-Trent. At the time of the study head-injured patients were admitted for overnight observation if they were confused or unconscious, vomiting or complaining of double vision at the time of assessment. History of very brief LOC or amnesia may lead to admission if there was no responsible relative to provide with head injury instructions. During the study period a head injury clinic was established at which patients admitted as a result of minor head injury were followed up. The clinic offered the opportunity to examine the natural history of post-concussive symptoms and the way this differed in patients who attended as a result of head injury but were not admitted.

\section{METHODS}

Two groups of patients were included in the study. Both had attended the A\&E Department of the North Staffordshire Royal Infirmary following minor head 
K. Barrett et al. injury. For the purposes of this study, minor head injury was defined as head injury which resulted in a period of unconsciousness or confusion/amnesia of less than $6 \mathrm{~h}$.

The first subject group was drawn from patients who had been admitted for observation following minor head injury. During a 3-month period patients over the age of 10 years who were admitted for overnight observation as a result of minor head injury were given a follow-up appointment 2 weeks after admission. At this interview a screening questionnaire developed by the authors formed the basis of a structured clinical interview conducted by the clinic nurse. The questionnaire included an open question on symptoms experienced since injury, followed by 30 direct questions on common post-head injury and other neuropsychiatric and neurological symptoms. This symptom check-list is included as Appendix 1. Data were obtained on basic demographic variables, cause, nature and immediate outcome of injury (corroborated by A\&E Department notes), past medical history and inquiry about return to work and leisure pursuits. Any patient reporting unexplained LOC since the injury, worsening headache or evidence of marked cognitive decline was referred immediately to one of the physicians involved in the study. During the study period only one such patient was so referred. All other patients were offered reassurance that their symptoms were not unusual and would be very likely to resolve. They were then given a further clinic appointment date for 12 weeks after their injury. At the 12-week interview the questions on symptoms, return to work and leisure activities were repeated.

The second subject group was drawn from patients who attended the A\&E Department as a result of minor head injury but were not admitted. Over a period of 1 month every third patient over the age of 10 years who was discharged from the A\&E Department with a diagnosis of minor head injury was sent a letter and postal questionnaire 2 weeks after their injury. The questionnaire was an abbreviated version of that administered in the head injury clinic. It retained the open and all the direct questions on symptoms and return to activities and a pre-paid and addressed envelope was included. Patients who returned a completed questionnaire were sent a further questionnaire covering the same areas 12 weeks after their injury.

This paper focuses upon symptomatology in patients who either attended the out-patient clinics or returned postal questionnaires at 2 and 12 weeks.

\section{RESULTS}

During the study period 48 patients who had been admitted to hospital for overnight observation were given out-patient appointments. All had been rendered unconscious by their injury. Thirty attended their clinic appointment 2 weeks after injury and 24 of them attended a second appointment 12 weeks after injury. Two- and 12-week data for those 24 patients will be presented.

A total of 171 patients discharged directly from the A\&E Department, with a recorded diagnosis of head injury, were sent postal questionnaires 2 weeks after injury. Eighty-seven questionnaires were returned completed. Fifty-eight of them returned a second questionnaire 12 weeks after injury. All had attended the A\&E Department because of symptoms arising directly from the head injury. The A\&E Department notes recorded no specific symptoms at the time of initial assessment in 22 (37.9\%) patients, laceration in $23(39.7 \%)$ patients, report of brief LOC and laceration in eight (13.8\%) patients, LOC alone in four $(6.9 \%)$ patients and LOC and brief confusion in one $(1.7 \%)$ patient. Two- and 12 week data for the 58 patients will be presented. A patient reporting LOC actually reports a period of amnesia which is presumed or confirmed by others to be caused as a result of LOC. It was not clear in the notes whether LOC was reported by the patient or a relative/observer.

Table 1 gives the age and sex distribution of the two groups, and the cause of injury. The number of symptoms experienced by the two groups at 2 and 12 weeks are presented in Table 2 . In the clinic group, one patient had more symptoms at 12 weeks than at 2 weeks, three patients had an equal number and 20 had fewer symptoms. In the postal questionnaire group, 12 patients had more symptoms at 12 weeks, 19 had an equal number and 28 had fewer symptoms. At 2 weeks the median number of symptoms reported (the symptom score) was 5.5 for the clinic group and $\mathbf{3 . 0}$ for the postal questionnaire group. Symptom scores between the two groups at 2 weeks were compared using the Mann-Whitney $U$-test and were found not to be significantly different $(P<0.35)$. The score differences in individual patients between 2 and 12 weeks (the change in score) were compared using the Mann-Whitney $U$-test. The average reduction in the symptom score in the clinic group was 4.0, but the average reduction in the postal questionnaire group was zero. This figure arose because of the large number of patients in that group who had more symptoms 
head injury
Clinic group $(n=24)$

Questionnaire group $(n=58)$

\begin{tabular}{lcc}
\hline Males & 15 & 44 \\
Females & 9 & 14 \\
& & \\
Age range (years) & $11.6-88.2$ & $11.7-77.1$ \\
Mean age (years) & 37.9 & 37.2 \\
& & \\
Cause of injury (\%) & & \\
Road traffic & & \\
accident & 37.0 & 20.3 \\
Industrial & 20.8 & 20.3 \\
Domestic & 20.8 & 27.1 \\
Assault & 8.3 & 10.2 \\
Sport & 8.3 & 15.3 \\
Others & 4.3 & 6.8 \\
\hline
\end{tabular}

\begin{tabular}{ccccc}
\hline \multirow{2}{*}{ Number of symptoms } & \multicolumn{2}{c}{ Clinic group } & \multicolumn{2}{c}{ Questionnaire group } \\
& 2 weeks & 12 weeks & 2 weeks & 12 weeks \\
\hline 0 & 4 & 12 & 17 & 23 \\
1 & 3 & 3 & 5 & 4 \\
2 & 0 & 2 & 4 & 4 \\
3 & 2 & 1 & 5 & 3 \\
4 & 0 & 2 & 3 & 3 \\
5 & 3 & 1 & 3 & 2 \\
6 & 1 & 0 & 1 & 1 \\
7 & 3 & 0 & 1 & 4 \\
8 & 1 & 0 & 2 & 3 \\
9 & 0 & 1 & 3 & 2 \\
10 & 0 & 0 & 3 & 0 \\
$<10$ & 7 & 2 & 12 & 10 \\
Range & $0-16$ & $0-14$ & $0-28$ & $0-24$ \\
Median & 5.5 & 0.5 & 3.0 & 2.0 \\
\hline
\end{tabular}

Table 1. Age, sex and cause of injury

Table 2. Number of symptoms reported

at 12 weeks than at 2 weeks. The fall in symptom scores was significantly greater in the clinic group $(P<0.0003)$.

The rank ordering of symptoms in the two groups (i.e. the percentage of patients experiencing particular symptoms) at 2 and 12 weeks are presented in Table 3. Spearman's Rank Correlation Coefficients were calculated. Rank ordering of symptoms at 2 and at 12 weeks, were significantly correlated $(r=0.51, P<0.03$ and $r=0.74, P<0.01$ respectively). In other words the clinical syndrome was similar whether or not the patient had sufficient symptoms or signs at initial assessment to lead to admission. Reduced vitality, irritability, headache and dizziness were the most common symptoms in the clinic group and neck-ache, irritability, reduced vitality and headache were the most common symptoms in the postal questionnaire group.

\section{DISCUSSION}

This study demonstrates that the symptoms experienced after minor head injury are similar regardless of whether immediate symptoms or signs lead to overnight admission. There were, however, some interesting differences. The most frequently reported symptom in patients who were not admitted was neck-ache, but this was only the fifth most common symptom in admitted patients. The actual percentage reporting neck-ache was similar in the two groups at 2 weeks (44\% in non-admitted patients and $41.7 \%$ in admitted patients). However, neck-ache proved to be more persistent in the non-admitted group. 
K. Barrett et al.

\begin{tabular}{|c|c|c|c|}
\hline Clinic group $(n=24)$ & $\%$ & Questionnaire group $(n=58)$ & $\%$ \\
\hline \multicolumn{4}{|l|}{ At 2 weeks post-injury } \\
\hline Reduced vitality & 58.3 & Neck-ache & 44.0 \\
\hline Irritability & 58.3 & Irritability & 35.6 \\
\hline Headache & 45.8 & Reduced vitality & 35.6 \\
\hline Dizziness & 45.8 & Headache & 33.9 \\
\hline Neck-ache & 41.7 & Impaired memory & 30.5 \\
\hline Impaired concentration & 37.5 & Reduced interest & 27.1 \\
\hline Impaired sleep & 37.5 & Impaired concentration & 25.4 \\
\hline Noise intolerance & 33.3 & Light intolerance & 25.4 \\
\hline Reduced appetite & 29.2 & Depressed mood & 25.4 \\
\hline Reduced interest & 29.2 & Breathlessness & 23.7 \\
\hline Anxiety & 29.2 & Impaired sleep & 23.7 \\
\hline Aggression & 25.0 & Dizziness & 20.2 \\
\hline Depressed mood & 20.8 & Anxiety & 20.3 \\
\hline Impaired memory & 16.7 & Feelings of unreality & 20.3 \\
\hline Clumsiness & 16.7 & Difficulty expressing & 18.6 \\
\hline Weakness & 16.7 & Aggression & 18.6 \\
\hline Paraesthesia & 16.7 & Paraesthesia & 18.6 \\
\hline Light intolerance & 12.5 & Weakness & 18.6 \\
\hline Blurred vision & 12.5 & Numbness & 18.6 \\
\hline \multicolumn{4}{|c|}{ (Spearman's Rank Correlation Coefficient: $r=0.51, P<0.027$ ) } \\
\hline \multicolumn{4}{|l|}{ At 12 weeks post-injury } \\
\hline Reduced vitality & 29.2 & Neck-ache & 30.5 \\
\hline Headache & 20.8 & Impaired sleep & 27.1 \\
\hline Irritability & 20.8 & Impaired memory & 25.4 \\
\hline Anxiety & 20.8 & Breathlessness & 25.4 \\
\hline Impaired concentration & 16.7 & Impaired concentration & 22.4 \\
\hline Impaired memory & 16.7 & Feelings of unreality & 22.4 \\
\hline Neck-ache & 16.7 & Reduced vitality & 22.0 \\
\hline Depressed mood & 12.5 & Anxiety & 22.0 \\
\hline Dizziness & 12.5 & Headache & 20.3 \\
\hline Aggression & 8.3 & Reduced appetite & 18.6 \\
\hline Reduced interest & 8.3 & Paraesthesia & 16.9 \\
\hline Impaired sleep & 8.3 & Difficulty expressing & 16.9 \\
\hline Light intolerance & 4.2 & Aggression & 16.9 \\
\hline Blurred vision & 4.2 & Dizziness & 16.9 \\
\hline Numbness & 4.2 & Reduced interest & 15.2 \\
\hline Paraesthesia & 4.2 & Depressed mood & 15.2 \\
\hline Weakness & 4.2 & Blurred vision & 11.9 \\
\hline Clumsiness & 4.2 & Weakness & 11.9 \\
\hline \multicolumn{4}{|c|}{ (Spearman's Rank Correlation Coefficient: $r=0.74, P<0.001$ ) } \\
\hline
\end{tabular}

Table 3. Rank order of symptoms 
Sequelae of minor head injury irritability, headache and dizziness. However, dizziness was more than twice as common in the admitted group (45.8\% as compared with $20.3 \%$ ) which could also relate to there having been more brainstem trauma in these patients.

Despite these differences the syndromes in the two groups were significantly correlated, particularly at 3 months. This similarity was not unexpected. What was unexpected was the finding that the reduction in symptoms at 3 months was significantly greater in the admitted than the non-admitted group. There are several possible explanations for this: (1) losing consciousness briefly may lead to a better prognosis, possibly because this may be associated with less soft-tissue damage to the neck; (2) offering out-patient follow-up and reassurance to minor head injury patients may lead to more rapid resolution of symptoms. A previous study ${ }^{5}$ examined the effects of telephone follow-up counselling on the sequelae of minor head injury. Patients who received telephone counselling were found to return to work significantly earlier than patients who were not counselled; (3) overnight admission to hospital after minor head injury could have similar beneficial effects; and (4) the findings could simply be a function of sample bias or the method of eliciting symptoms. Patients responding to the postal questionnaire, particularly 12 weeks after injury, may be those with the most enduring troubling symptoms, or those most inclined to complain. It is notable that 12 patients in the postal and questionnaire group had more symptoms at 12 weeks than at 2 weeks. The development of new post-concussive symptoms some time after injury has however been reported previously ${ }^{1}$ and may thus be a relatively common event in the natural history of the syndrome which merits further investigation. A similar selection bias would, in any event, also apply to patients who returned to the clinic. Indeed, it could be argued that clinic attenders may be a more biased group, given that returning to an outpatient clinic requires more effort and inconvenience than completing and posting a questionnaire. A significant methodological flaw of the present study is the use of a postal questionnaire in one group and a standardized interview (albeit using the same questions) in the other. A postal questionnaire follow-up of admitted patients would provide more accurate comparison data. In addition, a symptom check-list applied on initial presentation at A\&E would have enabled patients with the most trivial injuries to be excluded. Such trivial injuries (leading, for example, to laceration without any other immediate or longer term sequelae) may account for the relatively poor initial response to the postal questionnaire.

Whatever the explanation for the significantly greater resolution of symptoms in the clinic group the findings of this pilot study warrant further investigation. The study also suggests that there may be a significant overlap in the sequelae of minor neck ('whiplash') injuries and head-injured patients, particularly those who do not experience LOC. The frequency of supposedly 'post-concussive' symptoms in whiplash patients has been highlighted recently ${ }^{6}$ and deserves further study, as does the impact of different aftercare strategies on the resolution of symptoms and disability in both groups.

\section{ACKNOWLEDGEMENTS}

One of the authors $(A B)$ was funded during the study by a grant provided by the Nuffield Provincial Hospitals Trust.

\section{REFERENCES}

1. Rutherford W.H., Merrett J.D. \& McDonald J.R. (1979) Symptoms at one year following concussion from minor head injuries. Injury 10, 225-230.

2. Cartilidge N.E.F. \& Shaw D.A. (1981) The postconcussional syndrome. In: Head Injury. W.B., Saunders \& Co Ltd, London.

3. Binder L.M. (1986) Persisting symptoms after mild head injury: a review of post-concussion syndrome. Journal of Clinical and Experimental Neuropsychology 8, $323-346$.

4. Dikmen S., McLean A. \& Temkin A. (1986) Neuropsychological and psychosocial consequences of minor head injury. Journal of Neurology, Neurosurgery and Psychiatry 49, 1227-1232.

5. Hinkle J.L., Alves W.M., Rimell R.W. \& Jane J.A. (1986) Restoring social competence in minor headinjured patients. Journal of Neuroscience Nursing 18 , 268-271.

6. Radanov B.P., Stefano G.D., Schnidrig A. \& Ballinari P. (1991) Role of psychosocial stress in recovery from common whiplash. The Lancet 338, 712-715.

(Appendix 1 is continued on p. 84) 


\section{K. Barrett et al. APPENDIX 1}

\section{Symptom checklist}

How has your vitality been? (Have you felt as energetic as usual?)

Have you suffered from headaches?

Have you experienced any dizziness?

Do bright lights trouble you?

Have you experienced any double or blurred vision?

Do loud noises trouble you?

Have you felt more irritable or touchy than usual?

How is your temper? Have you been any more aggressive than usual?

Have you felt more sad or depressed than usual?

Have you felt more anxious or worried than usual?

Have you suffered any aching of your neck or shoulders?

Have you developed any new fears or phobias?

Have there been any times when you felt strange or unreal?

Have you had any problems sleeping?

Has your appetite altered?

Have you had your usual interest in things?

Has there been any change in your sexual interest?

Have you had any difficulty concentrating?

Have you had any problem remembering things?

Have you had any numbness (arms, face, legs, trunk)?

Any pins or needles or other add sensations?

Have you had any weakness or your arms and legs?

Have you been any more clumsy than usual?

Have you had any sudden jerking or your arms, legs or face?

Has there been any change in your sense of smell or taste?

Have you lost consciousness at all: had any blackouts?

Any difficulty expressing yourself, finding the words you want?

Has your speech been slurred at all?

Have there been times when you have felt suddenly breathless or felt your heart beating strongly?

Any other bad or unpleasant experiences I have not covered? 